\title{
$\alpha$-Lipoic Acid Protects Against Ischemia-Reperfusion Injury in Simultaneous Kidney-Pancreas Transplantation
}

Nella Ambrosi, BSc, ${ }^{1}$ Victoria Arrosagaray, MD, ${ }^{2}$ Diego Guerrieri, PhD, ${ }^{1}$ Pablo D. Uva, MD, ${ }^{2}$ Jorgelina Petroni, MD, ${ }^{2}$ Mónica Buonpensiere Herrera, MD, ${ }^{2}$ Juan L. lovanna, PhD, ${ }^{3}$ Luis León, MD, ${ }^{2}$ Claudio Incardona, MD, ${ }^{4}$ $\mathrm{H}$. Eduardo Chuluyan, MD, ${ }^{1}$ and Domingo H. Casadei, $\mathrm{MD}^{2}$

Background. Multiple factors have been implicated in the process of ischemia-reperfusion injury (IRI) in organ transplantation. Among these factors, oxidative damage seems to initiate the injury. $\alpha$-lipoic acid (ALA) is a potent antioxidant that is used in patients with diabetic polyneuropathy. The aim of the present study was to determine the effect of ALA in patients undergoing simultaneous kidney-pancreas transplant by evaluating the functional recovery of the graft and biochemical markers of IRI. Methods. Twentysix patients were included in the following groups: (i) untreated control; (ii) donor and recipient (DR) ALA-treated, in which ALA was administered both to the deceased donor and to the recipients; and (iii) recipient ALA-treated group. The expression of inflammatory genes, as observed in biopsies taken at the end of surgery, as well as the serum cytokines, secretory leukocyte protease inhibitor, regenerating islet-derived protein 3//pancreatitis-associated protein, amylase, lipase, glucose, and creatinine levels were quantified as markers of organ function. Results. The DR group showed high levels of TGF $\beta$ and low levels of C3 and TNF $\alpha$ in the kidneys, whereas high levels of C3 and heme oxygenase were identified in pancreas biopsies. Decreases in serum IL-8, IL-6, secretory leukocyte protease inhibitor, and regenerating islet-derived protein $3 \beta /$ pancreatitis-associated protein were observed after surgery in the DR group. Serum lipase and amylase were lower in the DR group than in the control and recipient groups. Early kidney dysfunction and clinical pancreatitis were higher in the control group than in either treatment group. Conclusions. These results show that ALA preconditioning is capable of reducing inflammatory markers while decreasing early kidney dysfunction and clinical posttransplant pancreatitis.

(Transplantation 2015;00: 00-00)

\begin{abstract}
idney ischemia-reperfusion injury (IRI) is a coordinated process leading to delayed graft function and reduced long-term graft survival of the transplanted organ. Several factors, originating mainly from endothelial injury, inflammation, and recruited leukocytes, seem to be involved in the pathogenesis of IRI. Among them, reactive oxygen species (ROS), such as superoxide radical, hydrogen peroxide, and hydroxyl radicals, play a significant role in oxidative damage of the tissue. ${ }^{1,2}$ In fact, healthy kidneys generate small amounts of ROS in the course of renal oxidative metabolism.
\end{abstract}

\footnotetext{
Received 23 June 2015. Revision received 1 September 2015.

Accepted 12 September 2015.

${ }^{1}$ CEFYBO-CONICET, Facultad de Medicina, Universidad de Buenos Aires, Buenos Aires, Argentina.

${ }^{2}$ Instituto de Nefrología de Buenos Aires, Buenos Aires, Argentina.

${ }^{3}$ Centre de Recherche en Cancérologie de Marseille (CRCM), Aix-Marseille Université and Institut Paoli-Calmettes, Parc Scientifique et Technologique de Luminy, Marseille, France.

${ }^{4}$ GADOR SA, Buenos Aires, Argentina.
}

This work was partially supported by CONICET PIP1001, UBACYT2014-2017 and Fundación GADOR S.A.

The other authors declare no conflicts of interest.

H.E.C. and D.H.C. contributed equally to the work.

C.I. is an employee of GADOR Argentina S.A.

N.A. participated in the performance of the research and data analysis. V.A. participated in the performance of the research. D.G. participated in the
However, under an injury, the production of ROS is increased by parenchyma cells or inflammatory infiltrating cells and contributes to acute or chronic renal injury. ${ }^{3,4}$ Therefore, there is special interest in testing agents with antioxidant properties that could possibly reduce the deleterious effects of ROS, and thus, the effect of the ischemia and reperfusion on the grafts.

$\alpha$-lipoic acid (ALA) is a fat- and water-soluble, naturally occurring, potent antioxidant. It has the ability to regenerate other factors, such as vitamins $\mathrm{C}$ and $\mathrm{E}$, in addition to raising glutathione intracellularly. Several studies have documented a beneficial therapeutic effect of ALA, benefits encompassing

performance of the research. P.U. participated in data analysis and writing of the article. J.P. participated in the performance of the research. M.B.H. participated in the performance of the research. J.I. participated in the performance of the research and contributed new reagents or analytic tools. L.L. participated in the performance of the research. C.I. participated in the writing of the manuscript and in research design. E.C. participated in the data analysis, Contributed with new reagents or analytic tools, and participated in the writing of the article and in research design. H.E.C. participated in data analysis, contributed new reagents or analytic tools, participated in the writing of the article and in research design. D.H.C. participated in data analysis, writing of the article, and research design.

Correspondence: H. Eduardo Chuluyan, MD, Facultad de Medicina, Universidad de Buenos Aires, CEFYBO-CONICET, Paraguay 2155, Piso 16, C1121ABG, Buenos Aires, Argentina. (echuluyan@gmail.com); (echuluyan@fmed.uba.ar).

Copyright (C) 2015 Wolters Kluwer Health, Inc. All rights reserved.

ISSN: 0041-1337/15/0000-00

DOI: $10.1097 /$ TP.0000000000000981 
diseases, such as diabetes, atherosclerosis, neurodegenerative diseases, and AIDS, among others. ${ }^{5}$ Specifically in IRI, ALA has been shown to be protective in several rat models, such as intestinal reperfusion, ${ }^{6}$ isolated rat heart and hindlimbs, ${ }^{7}$ hepatic IRI, ${ }^{8}$ acute pancreatitis, ${ }^{9}$ and kidney. ${ }^{10}$ In these later studies, the intraperitoneal administration of $100 \mathrm{mg} / \mathrm{kg}$ of ALA to rats subjected to 45 minutes of ischemia by renal pedicle occlusion was able to reverse the deleterious effect of the ischemia, such as the increases in serum creatinine, IL-1 $\beta$, IL-6, and TNF $\alpha$, among others. ${ }^{11}$ Also in rats, the intraperitoneal administration of ALA prevented the dysregulation of aquaporins and sodium transporters that is observed after kidney reperfusion ${ }^{10}$ and attenuated the increased expression of endothelin-1, which leads to kidney dysfunction and injury caused by IRI. ${ }^{12}$ Interestingly, in these studies, the administration of this high dose of ALA (100 mg/kg) was done before ischemia and immediately before the reperfusion period.

The beneficial effect of ALA has also been observed in humans. For example, ALA reduced hepatic IRI after inflow occlusion and liver resection. ${ }^{13}$ However, the most significant proven therapeutic effect of ALA in humans is on many of the complications induced by diabetes, including polyneuropathy and cataract formation. ${ }^{14}$ In fact, intravenous (IV) and oral forms of ALA are approved for treatment of diabetic sensorimotor polyneuropathy in several countries around the world, including Argentina. The huge amount of work done in different ischemia-reperfusion animal models mentioned above in addition to the beneficial effect observed in humans and the lack of serious adverse effects justify the putative use of ALA in transplant settings, where the production and effect of ROS have proved to be detrimental for grafts survival.

Because diabetes patients with chronic kidney disease experience excessive morbidity and mortality, simultaneous kidney-pancreas (SKP) transplantation is the treatment of choice for patients diagnosed with type 1 diabetes mellitus with end-stage chronic renal disease. ${ }^{15,16}$ Many of these patients suffer from diabetic sensorimotor polyneuropathy. Therefore, ALA administration by the time of the transplant may be beneficial not only for diabetic complications in the host but also for IRI's effects on the grafts from the organ procurement until the reperfusion in the host.

Therefore, the aim of the present study was to determine the effect of ALA in patients undergoing SKP transplantation, evaluating functional recovery of graft and biochemical markers of IRI.

\section{MATERIALS AND METHODS}

\section{Patient Selection}

The clinical and research activities being reported are consistent with the Principles of the Declarations of Helsinki and Istanbul, as outlined in the "Declaration of Istanbul on Organ Trafficking and Transplant Tourism." An institutional board and research committee approved this prospective study. Informed consent for the administration of ALA to deceased donor organs was unnecessary as stated in the IRB protocol, as allowance to preconditioning the donor already existed.

The study included 26 kidney-pancreas transplant patients (11 men and 15 women; age range, 21-57 years). Transplants were performed between April 28, 2011, and January 17, 2013, at the Instituto de Nefrología de Buenos Aires, Nephrology. Because both IV and oral forms of ALA are approved to mitigate the diabetic sensorimotor polyneuropathy in Argentina, all patients recruited for the study suffered from diabetic sensorimotor polyneuropathy. The recipient selection criteria were as follows: (1) age range, 18 to 65 years; and (2) received an SKP transplant. The patients received induction therapy consisting of thymoglobulin $(1.5 \mathrm{mg} / \mathrm{kg}$ for 5 days) and Solumedrol, and they were started on a triple immunosuppressive protocol (Tacrolimus with target level of 10 to $12 \mathrm{ng} / \mathrm{ml}$, prednisone tapered to $4 \mathrm{mg} / \mathrm{day}$, and mycophenolate sodium $1440 \mathrm{mg} /$ day).

All recipients included in the study had signs of sensorimotor polyneuropathy.

The patients were divided into 3 groups: (1) Control: received no ALA treatment; (2) ALA recipient (R)-treated: ALA (600 mg) was administered to the recipients only immediately before the surgical procedure; and (3) ALA DR-treated: ALA (600 mg) was administered to the deceased donor at the time of procurement and to the recipients immediately before the surgical procedure. The aim of ALA administration is to reduce the untoward effect of the ROS that are produced throughout the process known as IRI, which starts in the donor and continues in the recipient. This experimental design of comparing the ALA DR-treated group with the ALA R-treated group tends to unravel the impact of ROS that are produced during different steps of the IRI process. Thus, the treatment group was divided into groups 2 and 3 to address both the impact of ROS produced during different steps of the IRI process and the effect of donor preconditioning because in group 3, both donor and recipient were treated with ALA. The donor ALA preconditioning was performed just before the procurement procedure by IV drip $(20 \mathrm{mi}-$ nutes) of $600 \mathrm{mg}$ of ALA diluted in $250 \mathrm{~mL}$ saline at the beginning of the donor operation, which was approximately 90 to 120 minutes before clamping. The choice of donor dosage was based on previous work by Dünschede et $\mathrm{al}^{13}$ and Müller et al, ${ }^{17}$ who examined humans and rodents, respectively. Further, this dose has been approved by our local regulatory authorities to treat diabetic neuropathy. Administration of this antioxidant was considered part of the normal process of organ harvesting, and therefore, no further informed consent was necessary beyond that associated with organ donation. All organ procurements were performed by our team with the "no touch" technique, and organs were flushed with 2 L of aortic flush with University of Wisconsin solution. Portal flush for the liver was performed through a portal venotomy distal to the pancreas. The organs were then packed and stored in ice until transplantation. The ALApreconditioned organs and ALA IV administration were distributed according to simple random numbers enclosed in numbered and sealed envelopes, which were opened in the operating room only after the donor organ was accepted and before IV ALA administration.

Blood samples were obtained at the beginning and end of surgery, after the unclamping procedure, 12 hours after surgery, and every 1 or 2 days after the transplantation for at least 14 days or until discharge of the patient as part of the routine determination of analytes to assess kidney and pancreatic function. Furthermore, kidney and pancreatic biopsies were taken at the end of surgery to perform a real-time polymerase chain reaction (RT-PCR) study. The amount of biopsied tissue was not enough to simultaneously performed immunohistochemical staining. 
TABLE 1.

Demographic data of transplanted patients ${ }^{a}$

\begin{tabular}{|c|c|c|c|}
\hline & Control & DR & $\mathbf{R}$ \\
\hline & $(n=11)$ & $(n=7)$ & $(n=8)$ \\
\hline Male, $\%$ & 36.4 & 57.1 & 37.5 \\
\hline Recipient age, y & $35.4 \pm 6.8$ & $41.4 \pm 10.3$ & $41.5 \pm 10.9$ \\
\hline Recipient BMl, $\mathrm{kg} / \mathrm{m}^{2}$ & $22.4 \pm 2.7$ & $23.5 \pm 3.2$ & $25.7 \pm 3.4$ \\
\hline Time of dialysis, mo & $23.1 \pm 31.4$ & $28.7 \pm 18.1$ & $39.9 \pm 27.0$ \\
\hline \multicolumn{4}{|l|}{ CIT, h } \\
\hline Pancreas & $6: 82 \pm 2: 65$ & $7: 50 \pm 1: 10$ & $7: 92 \pm 1: 49$ \\
\hline Kidney & $8: 63 \pm 2.62$ & $8.87 \pm 1: 36$ & $9: 71 \pm 1: 29$ \\
\hline Donor age, y & $21 \pm 6.4$ & $27 \pm 11.7$ & $25 \pm 5.0$ \\
\hline
\end{tabular}

${ }^{a}$ Data represent the mean \pm SD for recipient age, recipient BMI, time of dialysis, CIT and donor age. There were no statistically significant differences among the groups.

CIT indicates cold ischemia time

\section{Serum Inflammatory Cytokines}

Serum levels of IL- 8 , IL-1 $\beta$, IL-6, IL-10, TNF- $\alpha$, and IL-12p70 were measured before and during the first 24-hour posttransplant surgery using the BD Cytometric Bead Array kit (BD Biosciences, San Jose, CA), following the manufacturer's instructions.

\section{Serum Secretory Leukocyte Protease Inhibitor And Regenerating Islet-Derived Protein} 3 及/Pancreatitis-Associated Protein Determination

Serum secretory leukocyte protease inhibitor (SLPI) was measured by sandwich ELISA (lower limit of detection, $0.31 \mathrm{ng} / \mathrm{mL}$ ) as previously described. ${ }^{18}$ Serum regenerating islet-derived protein $3 \beta$ (Reg $3 \beta)$ /pancreatitis-associated protein (PAP) was determined by sandwich ELISA following the manufacturer's instructions (PancrePAP, Dynabio, France). The serum Reg3ß/PAP concentration was measured before and during the first 24-hour posttransplant surgery and was expressed as nanograms per milliliter $(\mathrm{ng} / \mathrm{mL})$.

\section{RT-PCR Analysis}

Total RNA was isolated from the 19 (11 controls and 8 from DR group) fresh frozen biopsy samples using the RNeasy 5110 kit (Promega) according to the manufacturer's instructions. First-strand complementary DNA (cDNA) was synthesized from total RNA by reverse transcriptase (First Strand cDNA V3800 synthesis kit; Promega). The amplification and detection of cDNA in real-time quantitative PCR was performed using SYBER GreenER qPCR SuperMix Universal (Invitrogen, Grand Island, NY) according to the manufacturer's instructions. The instrument used for de qPCR was a Corbett Research Rotor-Gene 6000 (QIAGEN, Valencia, CA).

\section{Clinical Endpoints}

We assessed early kidney dysfunction and the development of clinical pancreatitis. Kidney dysfunction was defined as a decrease in creatinine of less than $70 \%$ of basal creatinine by day 7 after transplantation or the need for hemodialysis during the first week. Clinical pancreatitis was defined as a clinical status of abdominal distension, abdominal pain, pancreas graft swelling, and the need for pancreatic rest with total parenteral nutrition. This diagnosis was performed independent of this study by the clinicians who were in charge of the patient and was recorded in the patient posttransplant database.

We also assessed early posttransplant graft and patient survival at 3 months to determine the impact of these early events on hard endpoints.

\section{Statistical Analysis}

All statistics were analyzed in GraphPad Prism. Unpaired $t$ tests were used to compare means. A nonparametric test with Spearman rank correlation coefficient was used to analyze correlations. A $P$ value less than 0.05 was considered significant. Graphs were generated by GraphPad Prism (GraphPad, Inc., La Jolla, CA).

\section{RESULTS}

The patients comprised 11 men and 15 women, with a mean age of $38.9 \pm 9.1$ years, a BMI of $23.1 \pm 2.9 \mathrm{~kg} / \mathrm{m}^{2}$, an average of $21.7 \pm 5.8$ years with diabetes and a time on dialysis of $27.0 \pm 24.0$ months. Table 1 gives an overview of patient and donor demographics for each treatment group. Exocrine pancreas diversion was enteric and to the bladder in 21 and 5 patients, respectively.

First, we performed the RT-PCR analysis for C3, TNF $\alpha$, TGF $\beta$, and heme oxygenase (HMOX) from the pancreas and kidney biopsies from the control (untreated) and DR-treated groups. For the R-treated group, the time that serum ALA could be in contact with the allograft was very short because biopsies were taken at the end of surgery, whereas ALA was administered at the beginning. Therefore, the RT-PCR analysis for the R group was not performed because changes in the transcript levels are unlikely to occur within this short period (time from the unclamping till the end of the surgery).

Figure 1A shows that the kidney biopsies from the ALA DR-treated group expressed lower levels of C3 and TNF $\alpha$ and higher levels of TGF $\beta$ than those of the control biopsies $(P=0.003, P=0.0025$, and $P=0.0005$, respectively). Furthermore, the expression of HMOX-1 on these biopsies was not different from that of the untreated patients $(P=0.669)$. On the contrary, pancreas biopsies showed high expression of C3 $(P=0.0018)$ and HMOX-1 $(P<0.0001)$,

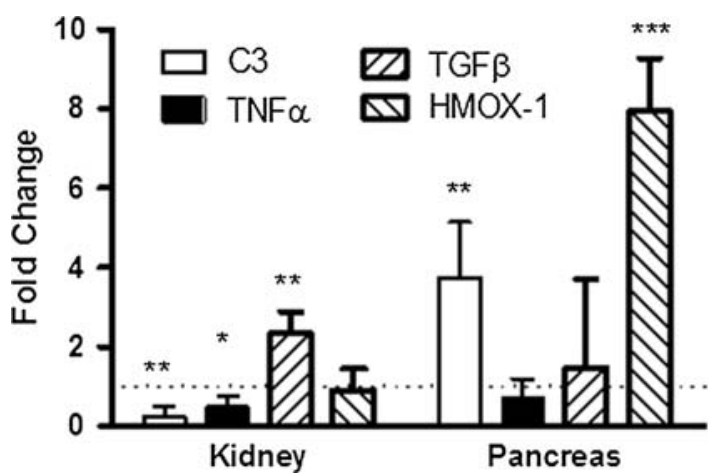

FIGURE 1. Real-time PCR results evaluating mRNA levels of some inflammatory mediators in SKP transplant patients. C3, TGF $\beta$, TNFa, and HMOX-1 were assayed by real-time PCR in kidney and pancreas RNA samples from untreated controls or ALA-treated donors. Biopsies were taken at the end of the transplant surgery. Values were normalized to the level of GADPH. Data represent the mean $\pm \mathrm{SD}$, and ${ }^{\star} P<0.05 ;{ }^{\star \star} P<0.01 ;{ }^{\star \star \star} P<0.001$. A 1-sample $t$ test was used to compare the untreated control group to a hypothetical value of 1 . 
the latter being more significant than C3. Unlike what was observed for kidneys, we did not observe significant differences in TNF $\alpha(P=0.171)$ or TGF $\beta(P=0.608)$ in the pancreas (Figure 1).

Next, we measured the serum levels of IL-8, IL-6, and IL-10 cytokines at the end of the surgery. Figure 2 shows that 24 hours after surgery, the untreated patients had higher serum levels of IL-8 $(P=0.028)$, IL-6 $(P=0.022)$, and IL-10 $(P=0.0016)$ than the pretransplant values. However, the serum levels of IL- 8 and IL- 6 for the ALA DR-treated group were similar to the pretransplant values and significantly lower than the untreated control group $(P=0.029$ for IL-8 and $P=0.039$ for IL-6; unpaired $t$-test). The ALA R-treated group showed a tendency to have lower levels of IL-6 and IL-10 but not of IL-8. However, the data observed in the R-treated group was not statistically significant compared with those of the untreated group (Figure 2).

Because pancreatitis causes a more than 200-fold increase in Reg3 $3 / \mathrm{PAP},{ }^{19}$ we next measured the serum levels of PAP. In all the groups analyzed, the serum level of PAP, measured at the end of the surgery (time, 0 hour after transplantation), increased relative to pretransplant values (Figure 3A). However, at 12 hours after transplantation, the level of PAP clearly decreased in the serum of ALA DR-treated groups
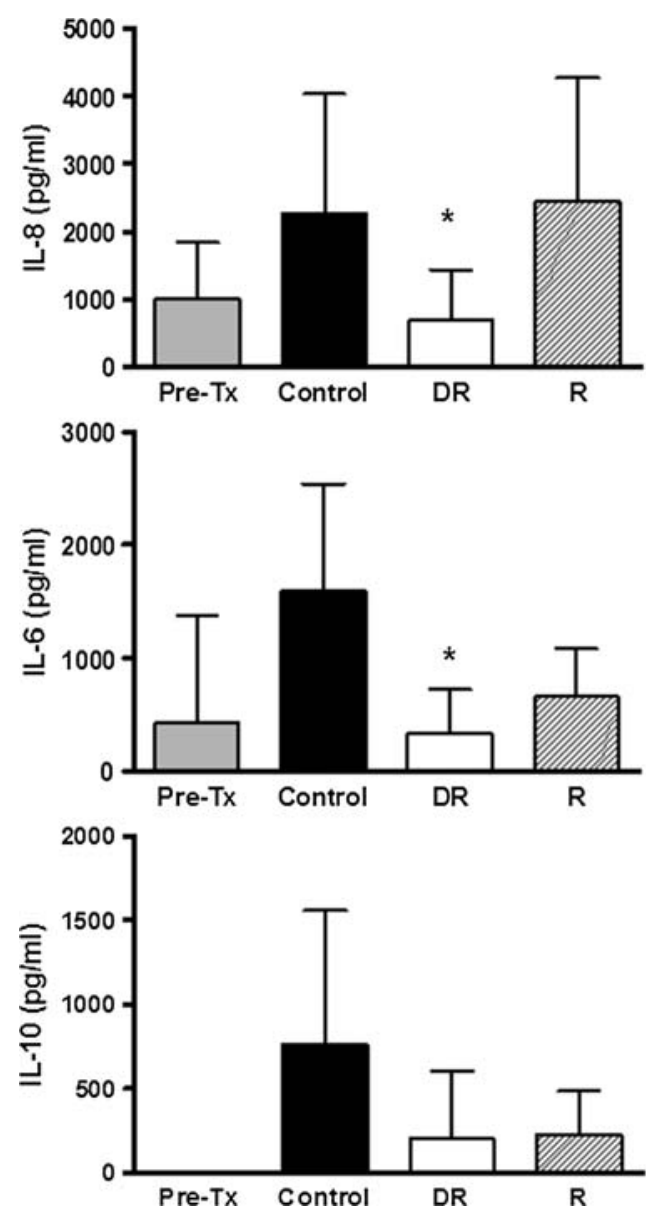

FIGURE 2. Serum levels of IL-8, IL-6, and IL-10. Serum cytokines were quantified by flow cytometry with a Cytometric Bead Array on samples taken before the surgery and 12 hours after the surgery. Data represent the mean $\pm \mathrm{SD}$. ${ }^{\star} P<0.05$. Unpaired $t$ test compared with control group.
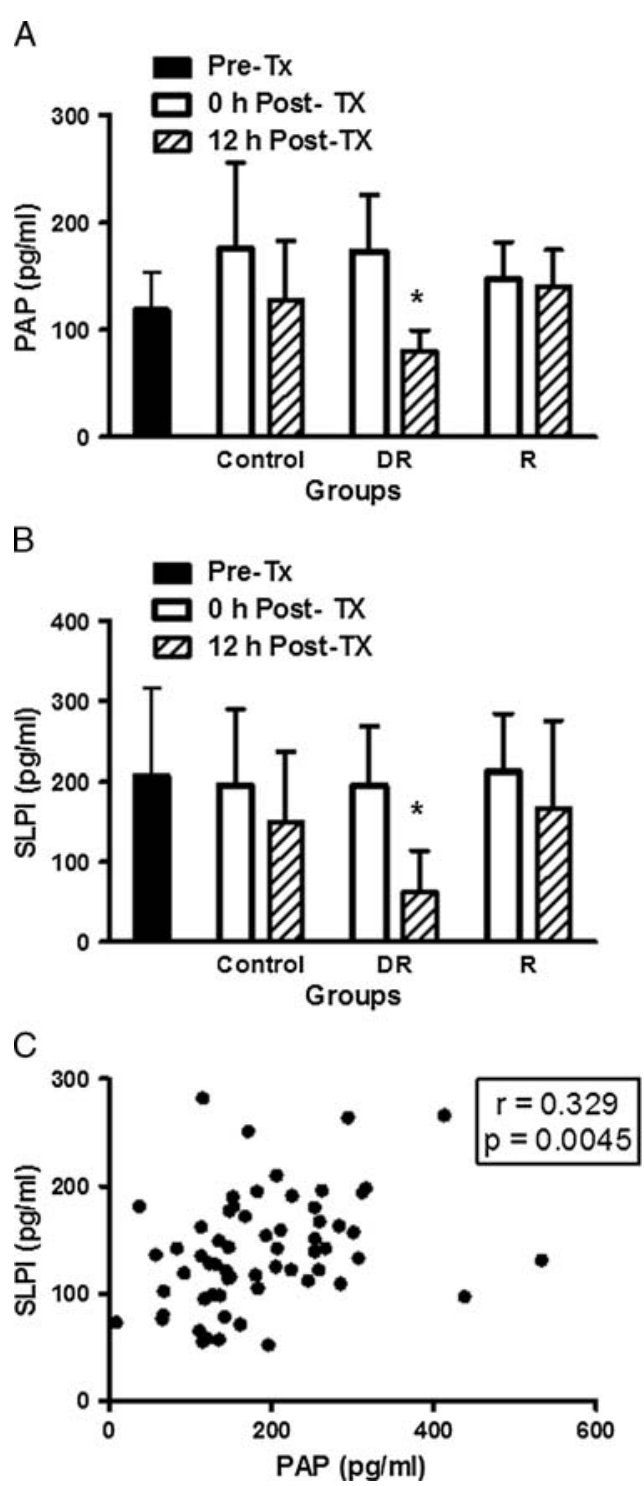

FIGURE 3. Serum levels of PAP and SLPI. Serum PAP (A) and SLPI (B) were quantified by sandwich ELISA on samples taken before the surgery, immediately after the surgery and 12 hours after the surgery. Data represent the mean $\pm \mathrm{SD}$ for $(\mathrm{A})$ and $(\mathrm{B}) .{ }^{\star} P<0.05$. Unpaired $t$ test compared with control group. C, Correlation between serum PAP and SLPI from all the serum samples analyzed (ie, before and 0 and 12 hours after transplantation). Coefficients $\left(R^{2}\right)$ and $P$ values are shown in the figure.

$(P=0.039$; Figure 3A, unpaired $t$ test compared with time 0 hour after transplantation). This effect was not observed in the untreated patients or the ALA R-treated patients. We next measured the level of SLPI, which is an alarm serine protease inhibitor with anti-inflammatory activity. Figure 3B shows that the levels of SLPI at the end of surgery (time, 0 hour after transplantation) were similar to the pretransplant values. By 12 hours after transplantation, the levels of SLPI had decreased in only the ALA DR-treated patients ( $P=0.044$, unpaired $t$-test), following the same pattern found for PAP. Moreover, we found a statistically significant direct correlation between PAP and SLPI serum plasma values (Figure 3C).

To determine the clinical significance of the inflammatory signature observed in the ALA DR- and R-treated groups, 
we next analyzed the graft function by assessing the serum levels of amylase, lipase, glucose, creatinine, and urea over a period of 14 days after the surgery. Figure 4A shows that the serum levels of amylase and lipase, but not of glucose, creatinine, or urea, were lower for the ALA DR-treated group than that for the control group at day 1 after transplantation. However, these differences were not statistically significant, probably due to the high variability found in the
A
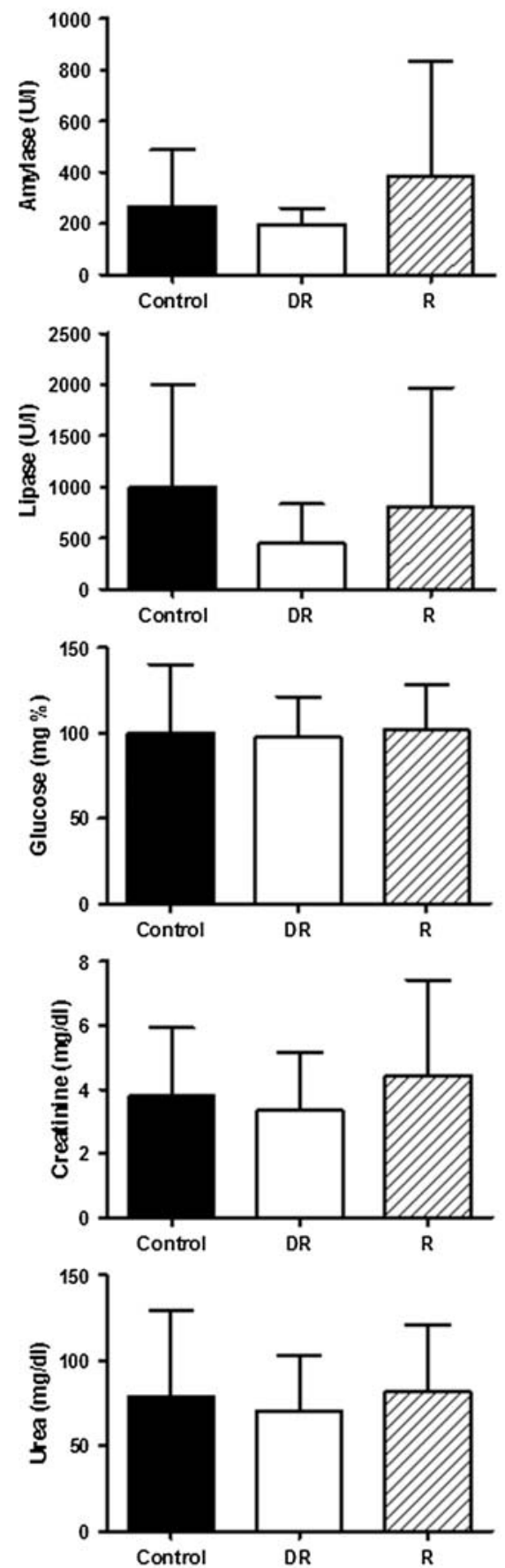

B
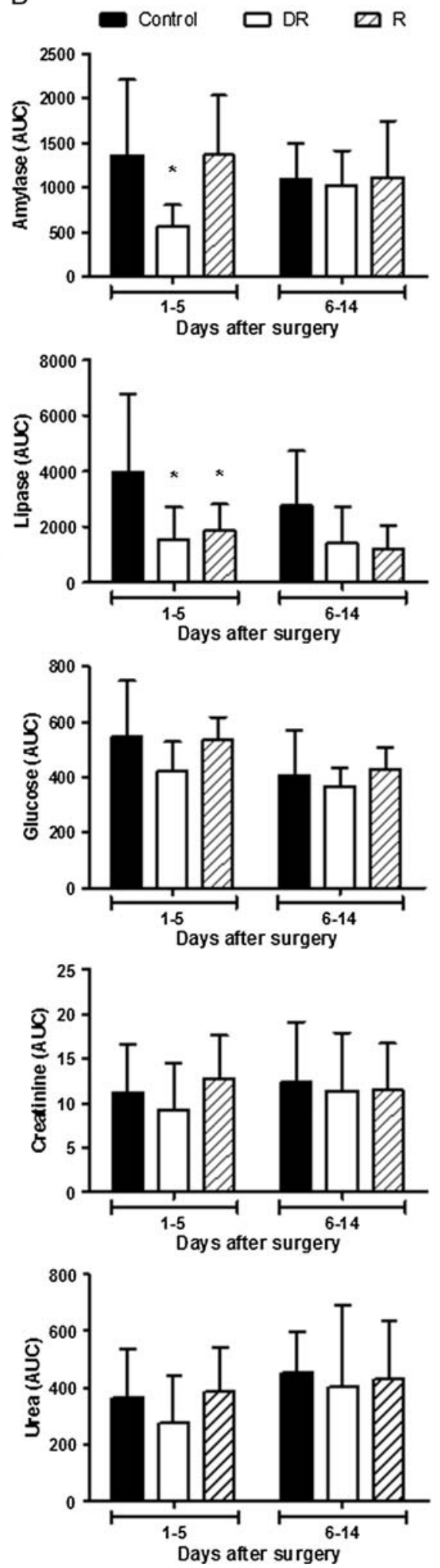

FIGURE 4. Serum levels of amylase, lipase, glucose, creatinine and urea. A, Serum levels of amylase, lipase, glucose, creatinine and urea at first day after the surgery. B, Area under the curve of serum values of biochemical markers analyzed during the first 5 days and 6 to 14 days after the surgery. Data represent the mean $\pm \mathrm{SD}$. ${ }^{*} P<0.05$. Unpaired $t$ test compared with the control group. 
serum values inside each group. Therefore, to avoid the high variability of values found in each group at the first day, we decided to analyze the values during the first 5 days for each patient. We then calculated the area under the curve for each patient and each analyzed parameter. Figure 4B shows that the DR-treated group had significantly lower levels of amylase $(P=0.015$, unpaired $t$ test $)$ and lipase $(P=0.039$, unpaired $t$ test) and that the R-treated group showed slightly lower levels of lipase than the untreated group $(P=0.066)$, confirming the tendency found in the values for the first day. With these analyses, we also observed a tendency toward lower levels of serum glucose, creatinine, and urea for patients in the ALA DR-treated group that was still not significantly different compared to those of the untreated patients. When the same analysis was performed using the serum values obtained from day 6 to 14 , we were unable to observe significant differences among the groups, likely because the serum values for the analytes in the control group began to decrease slightly. This was expected because all of the patients had recovered from the transplant and had been discharged from the center approximately after the second week of the surgery (Figure 4B).

Kidney dysfunction and clinical pancreatitis were also assessed as defined in the Methods section. There were 3 patients with early kidney dysfunction in the control group and 1 in both the ALA R- and ALA DR-treated groups (Table 2). We found 3 cases of pancreatitis in the control group, none in ALA R-treated group and 1 in ALA DR-treated group (Table 2). However, there were no differences in patient survival or in kidney and pancreas graft function during the first month after the transplantation. The follow-up of these patients at 3 months after transplantation revealed patient, kidney, and pancreas survival rates of $91 \%(10 / 11)$ in the controls and $100 \%$ in both ALA-treated groups (Table 2).

\section{DISCUSSION}

Reduced IRI in organ transplantation can decrease the incidence of organ loss caused by acute inflammation of the graft.

In the present article, we have shown that by targeting the production of ROS with an antioxidant drug, it was possible to reduce some inflammatory mediators and improve some clinical parameters. Thus, the results obtained herein reinforce the deleterious effect of ROS, as previously described, as one of the main mechanisms responsible for graft injury in solid organ transplantation. However, the effectiveness of antioxidant therapies has been questioned because a number of studies have not shown beneficial effects. In fact, in our study, treating the host with ALA at the time of the surgery

\section{TABLE 2.}

Short-Term Outcomes

\begin{tabular}{lccr}
\hline & Control & DR & R \\
\hline $\begin{array}{l}\text { \% Organ dysfunction } \\
\quad \text { Kidney dysfunction }\end{array}$ & 27.3 & 14.3 & 12.5 \\
$\quad \begin{array}{l}\text { Pancreatitis } \\
\text { \% Graft survival rate (at 3 mo) }\end{array}$ & 27.3 & 14.3 & 0 \\
$\quad$ Kidney & 91 & 100 & 100 \\
$\quad$ Pancreas & 91 & 100 & 100 \\
Patient survival rate (at 3 mo) & 91 & 100 & 100 \\
\hline
\end{tabular}

(R group) did not substantially improve most of the inflammatory or clinical parameters analyzed. However, a tendency toward improvement was observed in some clinical endpoints (such as pancreatitis and kidney dysfunction), even by treating only the recipients of the graft. This suggests that a better adjustment of the ALA doses or timing of the administration could improve graft outcome. In fact, preclinical animal studies have shown better results with high ALA doses $(100 \mathrm{mg} / \mathrm{kg})$ and have demonstrated that lower doses $(10 \mathrm{mg} / \mathrm{kg})$, such as that used in our study, are less effective. ${ }^{12}$ It is important to note that the doses used in our study were the ones that had been approved for diabetic neuropathy treatment by local regulatory authorities.

On the contrary, when the transplant is performed with organs from donors who had been previously treated with antioxidants, beneficial results were obtained for the inflammatory parameters that affected improved graft function (DR group). The multiple variables of the deceased donors may impact the quality of the graft, and this influence can be counteracted with ALA antioxidant-donor treatment. In fact, the RT-PCR analysis of inflammatory and antiinflammatory mediators in the kidney and pancreas derived from ALA-treated donors showed that ALA fostered decreased inflammation of the grafts, as indicated by the low expression of $\mathrm{C} 3$ and high expression of TGF $\beta$ in the kidney with a remarkably high expression of HMOX-1 in the pancreas. This result compares to the high C3 expression in the pancreas compared to untreated donor-derived organs. In this regard, it is interesting to note that it was recently proposed that the original classification of $\mathrm{C} 3 \mathrm{a}$ be changed from a "proinflammatory mediator" to an "inflammatory modulator," based on several anti-inflammatory facets found for C3a in vivo. ${ }^{20}$ The protective effect of HMOX-1 is not a surprise. Beneficial effects of this antioxidant molecule have been observed in several IRI animal models. ${ }^{21}$ However, unlike what has been observed in the kidney, where the expression of HMOX-1 was apparently associated with a poor outcome, ${ }^{22-24}$ in the pancreas, the upregulation of HMOX-1 may prevent fibrosis by inhibiting the proliferation of pancreatic stellate cells. ${ }^{25}$ The overall effect observed in biopsies from the DR group compared with the untreated group clearly indicates an effect of ALA on the donor.

Donor-preconditioning to reduce IRI is not a new concept. $^{26-29}$ Brain-dead rats treated with steroids or soluble P-selectin glycoprotein ligand had improved graft survival. ${ }^{30}$ Furthermore, in humans, the treatment of a brain-dead donor with steroids reduced the expression of proinflammatory cytokines. ${ }^{31}$ In fact, the DR ALA-treated group but not the ALA receptor-treated patient group in our study had a reduced expression of IL-8 and IL-6 (Figure 2), whereas IL-10, which is considered an anti-inflammatory cytokine, was unchanged. The efficacy of ALA in decreasing the levels of some cytokines was already described during extracorporeal circulation. ${ }^{32}$ However, we believe that by administering ALA immediately before ablation, ALA can protect the organ from the ROS produced by the ischemia due the ablation procedure, rather than from the storm of cytokines, which is highly likely to start well before the administration of ALA.

The PAP has been described in the pancreatic juice after transplantation ${ }^{33,34}$ and was found to be a good serum marker for pancreatic injury. ${ }^{35}$ We observed that the serum levels of PAP in patients who received grafts from an ALA-treated 
brain-dead donor were lower than those in patients who received untreated grafts and were treated with ALA at the time of surgery. The same pattern was observed for another alarm cationic nonglycosylated protein named SLPI, ${ }^{36}$ which has anti-inflammatory and microbicidal activity but has recently been suggested to be a biomarker of acute kidney injury. ${ }^{37}$ Indeed, a strong direct correlation was observed between PAP and SLPI (Figure 3C).

The ALA treatment clearly affects the levels of serum inflammatory markers. This effect was more marked for the ALA DR-treated group and, to a lesser extent, for the ALA $\mathrm{R}$-treated group relative to the untreated patients. However, we could see a trend toward a decrease in the incidence of early kidney dysfunction and clinical pancreatitis in the DR- and R-treated groups. The complexity of multiple pathologic mechanisms that causes kidney dysfunction and pancreatitis could explain the lack of an ideal association between all the inflammatory markers analyzed and the clinical outcome in the ALA R-treated group, as found for ALA DR-treated group.

Our findings for the cytokine profiles and levels of inflammation markers are stronger for the DR group than for the $\mathrm{R}$ group. These results suggest that the ROS produced by the donor during ablation might be more important than the ROS produced by the reperfusion. Thus, donor preconditioning with ALA plays a major protective role, as described herein.

It is probable that doses should be optimally adjusted to observe more significant results. In fact, in the R-treated group, we administered only 1 dose of ALA. A daily administration of ALA after surgery could perhaps improve the results because the values begin to increase after the fifth day to the level of the control group (Figure 4B). In general, ALA is a well-tolerated drug that has minor side effects. Nausea ${ }^{38}$ and itching have been reported with high doses (1 200-1 $800 \mathrm{mg}$ ) of ALA. ${ }^{39}$ However, recently, adverse effects on liver mitochondria have been reported with high IV doses. ${ }^{40}$ This high dose $(90-100 \mathrm{mg} / \mathrm{kg}$ ) extremely exceeded the dose used in our study (600 mg). In our study, we detected no side effects, such as those described in the studies mentioned above. However, an increased number of rejection episodes was observed for both the ALA DR- and R-treated groups (3 patients in each ALA-treated group) during the first posttransplant year (data not shown). However, there were no differences in early graft function. Therefore, these rejection episodes were related to other factors, such as adherence to the immunosuppressive treatment, the presence of donor-specific antibodies, and the presence of infection, among others. In fact, 2 of the 3 rejection episodes in the ALA DR group involved antibodymediated rejection, and 1 rejection episode was related to BK virus infection in the ALA R-treated group.

It is well known that brain death releases massive amounts of cytokines and growth factors and upregulates complement activation. ${ }^{38}$ To our knowledge, this report is the first to show that the use of an antioxidant may reduce inflammatory markers and improve some clinical parameters in human SKP transplantation. In fact, this preliminary study suggests that ALA preconditioning may be suitable for decreasing the inflammatory markers during the first days after SKP surgery, which may subsequently decrease the incidence of early kidney dysfunction and clinical graft pancreatitis. More studies need to be performed to fully optimize the pharmacology of this drug.

\section{ACKNOWLEDGMENTS}

This work was funded by UBACYT 2014-2017, CONICET PIP1001 and Fundación GADOR Argentina S.A. We thank the technical assistance of Mrs. Soledad Basualdo. Wiley editing service was used to edit the article.

\section{REFERENCES}

1. Sussman MS, Bulkley GB. Oxygen-derived free radicals in reperfusion injury. Methods Enzymol. 1990;186:711-723.

2. Yamanobe T, Okada F, luchi Y, et al. Deterioration of ischemia/reperfusioninduced acute renal failure in SOD1-deficient mice. Free Radic Res. 2007; 41:200-207.

3. Baud L, Ardaillou R. Reactive oxygen species: production and role in the kidney. Am J Physiol. 1986;251(5 Pt 2):F765-776.

4. Nath KA, Salahudeen AK. Induction of renal growth and injury in the intact rat kidney by dietary deficiency of antioxidants. J Clin Invest. 1990;86: 1179-1192.

5. Bilska A, Włodek L. Lipoic acid—-the drug of the future? Pharmacol Rep. 2005;57:570-577.

6. Roldán EJ, Kerzberg EM, Boveris A. Kinetics of chemoluminiscence of rat intestine during ischemia and reperfusion. Medicina (B Aires). 1989;49: 336-340.

7. Freisleben HJ. Lipoic acid reduces ischemia-reperfusion injury in animal models. Toxicology. 2000;148:159-171.

8. Dulundu E, Ozel Y, Topaloglu U, et al. Alpha-lipoic acid protects against hepatic ischemia-reperfusion injury in rats. Pharmacology. 2007;79:163-170.

9. Abdin AA, El-Hamid MA, El-Seoud SH, et al. Effect of pentoxifylline and/or alpha lipoic acid on experimentally induced acute pancreatitis. Eur $J$ Pharmacol. 2010;643:289-296.

10. Bae EH, Lee KS, Lee J, et al. Effects of alpha-lipoic acid on ischemiareperfusion-induced renal dysfunction in rats. Am J Physiol Renal Physiol. 2008;294:F272-280.

11. Sehirli $O$, Sener $E$, Cetinel $S$, et al. Alpha-lipoic acid protects against renal ischaemia-reperfusion injury in rats. Clin Exp Pharmacol Physiol. 2008;35: 249-255.

12. Takaoka M, Ohkita M, Kobayashi Y, et al. Protective effect of alpha-lipoic acid against ischaemic acute renal failure in rats. Clin Exp Pharmacol Physiol. 2002;29:189-194.

13. Dünschede F, Erbes K, Kircher A, et al. Reduction of ischemia reperfusion injury after liver resection and hepatic inflow occlusion by alpha-lipoic acid in humans. World J Gastroenterol. 2006;12:6812-6817.

14. Papanas N, Ziegler D. Efficacy of a-lipoic acid in diabetic neuropathy. Expert Opin Pharmacother. 2014;15:2721-2731.

15. Ojo AO, Meier-Kriesche HU, Hanson JA, et al. The impact of simultaneous pancreas-kidney transplantation on long-term patient survival. Transplantation. 2001;71:82-90.

16. Sollinger HW, Odorico JS, Becker YT, et al One thousand simultaneous pancreas-kidney transplants at a single center with 22-year follow-up. Ann Surg. 2009;250:618-630.

17. Müller C, Dünschede F, Koch E, et al. Alpha-lipoic acid preconditioning reduces ischemia-reperfusion injury of the rat liver via the PI3-kinase/Akt pathway. Am J Physiol Gastrointest Liver Physiol. 2003;285:G769-778.

18. Tateosian NL, Costa MJ, Guerrieri D, et al. Inflammatory mediators in exhaled breath condensate of healthy donors and exacerbated COPD patients. Cytokine. 2012;58:361-367.

19. lovanna JL, Keim V, Nordback I, et al. Serum levels of pancreatitisassociated protein as indicators of the course of acute pancreatitis. Multicentric Study Group on Acute Pancreatitis. Gastroenterology. 1994; 106:728-734.

20. Coulthard LG, Woodruff TM. Is the complement activation product C3a a proinflammatory molecule? Re-evaluating the evidence and the myth. $J$ Immunol. 2015;194:3542-3548.

21. Correa-Costa M, Amano MT, Câmara NO. Cytoprotection behind heme oxygenase-1 in renal diseases. World J Nephrol. 2012;1:4-11.

22. August C, Brockmann J, Vowinkel T, et al. Stress associated proteins metallothionein, HO-1 and HSP 70 in human zero-hour biopsies of transplanted kidneys. Virchows Arch. 2006;449:192-199.

23. Lemos FB, ljzermans JN, Zondervan PE, et al. Differential expression of heme oxygenase-1 and vascular endothelial growth factor in cadaveric and living donor kidneys after ischemia-reperfusion. J Am Soc Nephrol. 2003; 14:3278-3287.

24. Ollinger R, Kogler P, Biebl M, et al. Protein levels of heme oxygenase-1 during reperfusion in human kidney transplants with delayed graft function. Clin Transplant. 2008;22:418-423. 
25. Schwer Cl, Guerrero AM, Humar M, et al. Heme oxygenase- 1 inhibits the proliferation of pancreatic stellate cells by repression of the extracellular signal-regulated kinase1/2 pathway. J Pharmacol Exp Ther. 2008;327: 863-871.

26. Bos EM, Leuvenink HG, van Goor $\mathrm{H}$, et al. Kidney grafts from brain dead donors: inferior quality or opportunity for improvement? Kidney Int. 2007; 72:797-805.

27. Schnuelle P, Lorenz D, Mueller A, et al. Donor catecholamine use reduces acute allograft rejection and improves graft survival after cadaveric renal transplantation. Kidney Int. 1999;56:738-746.

28. Schnuelle P, Berger S, de Boer J, et al. Effects of catecholamine application to brain-dead donors on graft survival in solid organ transplantation. Transplantation. 2001;72:455-463.

29. Schnuelle P, Yard BA, Braun C, et al. Impact of donor dopamine on immediate graft function after kidney transplantation. Am J Transplant. 2004;4: 419-426.

30. Pratschke J, Kofla G, Wilhelm MJ, et al. Improvements in early behavior of rat kidney allografts after treatment of the brain-dead donor. Ann Surg. 2001;234:732-740.

31. Kuecuek O, Mantouvalou L, Klemz R, et al. Significant reduction of proinflammatory cytokines by treatment of the brain-dead donor. Transplant Proc. 2005;37:387-388
32. Uyar IS, Onal S, Akpinar MB, et al. Alpha lipoic acid attenuates inflammatory response during extracorporeal circulation. Cardiovasc J Afr. 2013; 24:322-326

33. Keim V, lovanna JL, Orelle B, et al. A novel exocrine protein associated with pancreas transplantation in humans. Gastroenterology. 1992;103:248-254.

34. Orelle B, Keim V, Masciotra L, et al. Human pancreatitis-associated protein. Messenger RNA cloning and expression in pancreatic diseases. $J$ Clin Invest. 1992:90:2284-2291.

35. van der Pij JW, Boonstra JG, Barthellemy S, et al. Pancreatitis-associated protein: a putative marker for pancreas graft rejection. Transplantation. 1997;63:995-1003.

36. Scott A, Weldon S, Taggart CC. SLPI and elafin: multifunctional antiproteases of the WFDC family. Biochem Soc Trans. 2011;39:1437-1440.

37. Wilflingseder J, Sunzenauer J, Toronyi E, et al. Molecular pathogenesis of post-transplant acute kidney injury: assessment of whole-genome mRNA and miRNA profiles. PLoS One. 2014;9:e104164

38. Ponticelli C. Ischaemia-reperfusion injury: a major protagonist in kidney transplantation. Nephrol Dial Transplant. 2014;29:1134-1140.

39. Koh EH, Lee WJ, Lee SA, et al. Effects of alpha-lipoic Acid on body weight in obese subjects. Am J Med. 2011;124:85 e81-88.

40. Vigil M, Berkson BM, Garcia AP. Adverse effects of high doses of intravenous alpha lipoic acid on liver mitochondria. Glob Adv Health Med. 2014;3:25-27. 